\title{
ESTUDIOS
}

Fernando Verdugo, S.J.

Universidad Alberto Hurtado

\section{Consideraciones preliminares sobre la credibilidad}

El tema del Seminario Interno son "Los desafíos actuales a la credibilidad del cristianismo". Estos serán abordados desde las distintas disciplinas teológicas. Para esta primera sesión se me han pedido algunas "Consideraciones preliminares sobre la credibilidad". Se trataría de reflexiones previas a un tratamiento más riguroso del tema (1), el cual tendrá lugar a lo largo del Seminario.

Sin duda que una reflexión teológico-fundamental sobre la credibilidad tendría que considerar un recorrido por las Sagradas Escrituras, fijándose en virtud de que algo o alguien es presentado en ellas como digno de fe. Podría uno preguntarse, por ejemplo: ¿cuáles son los elementos que contribuyen a la credibilidad de la fe judía y, luego, de la fe cristiana?, ¿a qué recursos se apela en el mundo bíblico para que los mensajes que porta sean acogidos por el oyente o lector?, ¿por qué, en definitiva, se ha de creer en Dios y vivir según sus designios? Una reflexión teológico-fundamental tendría que considerar también los criterios de credibilidad que la Iglesia ha ido destacando a lo largo de la historia. Si bien esos pasos son fundamentales, no creo que sean los únicos ni lo que a mí se me está pidiendo. Entiendo que dichos pasos serán dados por otros en el Seminario.

En esta presentación me detendré, en primer lugar, en lo que suele entenderse por "credibilidad" en términos generales y en algunos de los criterios por los cuales algo o alguien adquiere la calidad de creíble. Luego, en segundo lugar, pondré especial énfasis en la necesaria atención a lo cultural que debe prestar la teología interesada en asumir los desafíos que la sociedad le plantea, para así contribuir a la credibilidad del Evangelio.

\section{SIGNIFICADO Y CRITERIOS DE CREDIBILIDAD}

Por credibilidad se suele entender la "calidad de creíble" que se atribuye a una persona o institución (2); es decir, si una u otra es digna de fe o de confianza.

(1) Este es uno de los significados de "preliminar" que ofrece el Diccionario de la Lengua Española de la RAE, $21^{a}$ Edición: "que sirve de preámbulo o proemio para tratar sólidamente una materia".

(2) Véase el significado de "credibilidad" en el Diccionario de la Lengua Española de la RAE, $21^{\text {a }}$ Edición. 
Sabemos que la credibilidad se puede tener, luego perder y -generalmente con mayor dificultad- volver a recuperar. Últimamente hemos sido testigos de la pérdida de fe en importantes instituciones del país debido a algunos escándalos: casos de pedofilia o abuso de menores entre el clero han mellado la credibilidad de la Iglesia; el traspaso de información reservada del Banco Central a Inverlink o la usurpación y venta de bonos CORFO han afectado la confianza en el sistema financiero. Los ejemplos se podrían multiplicar. Pero en lugar de hacerlo, conviene destacar que la credibilidad no solo se atribuye a personas o instituciones. También las afirmaciones o mensajes que emiten personas o instituciones pueden ser más o menos creíbles.

¿Mediante qué criterios se mide la credibilidad de una persona, institución o mensaje? Son muchos y ciertamente varían según los contextos. Detengámonos en algunos.

Sabemos, por ejemplo, que en el mundo bajo el influjo de la cultura griega si una afirmación que se tiene por verdadera no aparece como razonable, normalmente pierde credibilidad y viceversa. Se comprende, entonces, que desde que el cristianismo comienza a expandirse por la cuenca del Mediterráneo "dar respuesta a todo el que pida razón de la esperanza" haya sido propuesta como una de las responsabilidades del creyente en Jesucristo (Cf. 1Pe 3,15).

Pero no solo incide en la credibilidad de una persona, de una institución o de una afirmación el que sea razonable. También es importante que sea significativa; es decir, portadora de sentido y relevante para la existencia personal y social. De ahí, por ejemplo, que muy pronto el kerigma cristiano haya explicitado su dimensión soteriológica: la vida, el mensaje y, por sobre todo, la muerte y resurrección de Jesús son una buena noticia que afecta -para bien- la existencia del ser humano (véase, por ej., Hch 3, 26; 5,31; 2 Tm 1,10; etc.). De ahí, también a modo de ejemplo, que la teología latinoamericana de la liberación haya intentado desde sus inicios explicitar qué implicaba la salvación de Cristo en un continente afligido por la pobreza y opresión (3).

Podemos agregar también que lo creíble está igualmente asociado con la belle$z a$, con lo que es capaz de producir gozo estético. La carta de Pedro antes citada nos enseña que lo "precioso ante Dios" ( $\pi 0 \lambda v \tau \varepsilon \lambda \varepsilon \varsigma)$ está en el corazón, en el interior y no en los adornos exteriores (1Pe 3,3-4). Por otra parte, creo que no es osado afirmar que la confesión de fe en Jesús como "la luz verdadera que ilumina a todo hombre" (Jn 1,9) o "resplandor de la gloria" de Dios (Heb. 1,3), en cuanto implica el uso de los sentidos, tiene también una connotación estética. Un ejemplo de la vida cotidiana puede ilustrar igualmente esta dimensión estética de la credibilidad. A quienes se nos ha encomendado el ministerio de la predicación, muchas veces hemos escuchado a la salida de una misa: "Linda su prédica, Padre". Estoy convencido de que casi siempre se trata de un mero cumplido. Pero a veces es posible captar que lo que hemos dicho y, sobre todo, el cómo lo hemos dicho, ha ayudado a que el mensaje transmitido sea acogido y se convierta en fuente de gozo y plenitud para el

(3) Véase, por ejemplo, Gustavo Gutiérrez, Teología de la Liberación. Perspectivas (CEP, Lima 1971), Ed. Sígueme, Salamanca 1990, 14ª ed. revisada y aumentada, especialmente el capítulo 9: "Liberación y Salvación". 
oyente. A esta misma dimensión estética de la credibilidad parece apuntar el libro La experiencia bíblica: gracia y ética, de Antonio Bentué. En efecto, en dicho libro Bentué nos acerca a la imagen auténtica del Dios que se revela en la historia de Israel y plenamente en Jesús: del Dios gratuito y gracioso, que goza haciendo el bien y que nos invita a participar no solo mística y éticamente, sino también estéticamente del mismo gozo (4).

Con lo dicho hasta ahora da la impresión de que el cristianismo tendría que ser intelectualmente razonable, éticamente significativo y estéticamente atractivo para estar dotado de credibilidad. Parece, entonces, que bastaría con responder a la siguiente pregunta: ¿cómo mostrar que creer en Jesucristo es razonable, bueno y hermoso para el ser humano? Sin embargo, la cosa no es tan sencilla. Hay otro nivel de preguntas de las que también habría que hacerse cargo. Por ejemplo: ¿cuáles son los paradigmas vigentes que gobiernan lo que se considera razonable, bueno o hermoso? ¿Son iguales esos paradigmas en las diversas culturas vigentes? Si no es así, ¿cómo presentar la fe cristiana de manera creíble a todas las gentes (Cf. Mt 28,19), inmersas en mundos culturales tan distintos?

Detrás de ese segundo nivel de preguntas subyace una convicción de fondo: aparte de los trascendentales arriba expuestos, es necesario poner atención en los condicionamientos culturales de la fe cristiana. La fe, al igual que toda experiencia humana, está culturalmente mediada. Tomar conciencia de ese hecho y asumir las consecuencias metodológicas, es indispensable para una teología que quiere poner atención a los desafíos actuales y contribuir así a la credibilidad del cristianismo. En el desarrollo de este punto estaría lo medular de mi reflexión.

\section{CONDICIONAMIENTOS CULTURALES DE LA FE CRISTIANA}

Es cierto que, como bien ha señalado Juan Pablo II en Fides et Ratio, "el proceso de encuentro y confrontación con las culturas es una experiencia que la Iglesia ha vivido desde los comienzos de la predicación del Evangelio. El mandato de Cristo a los discípulos de ir a todas partes 'hasta los confines de la tierra' (Hch, 1,8) para transmitir la verdad por Él revelada, permitió a la comunidad cristiana verificar bien pronto la universalidad del anuncio y los obstáculos derivados de la diversidad de las culturas" (70). Sin embargo, también es cierto que la captación más sistemática por parte de la Iglesia de lo que está detrás de lo cultural, de su impacto en la fe, de lo que implica para la evangelización y también para la convivencia humana, es algo bastante reciente. Y en este proceso de toma de conciencia, más que con la fílosofía, el diálogo con las ciencias sociales ha sido clave.

Si bien se dieron pasos importantes antes del Concilio Vaticano II (5), no hay duda de que este gran evento eclesial constituyó un giro decisivo en la preocupación

(4) Antonio BENTUÉ La experiencia bíblica: gracia y ética, PPC, Madrid 1997. He realizado una presentación del libro en Mensaje 465 (1997) 634.

(5) Por ejemplo, en la Comunicación de Pío XII en 1944 a los Sociedades de Ayuda a las Misiones Pontificias, se encuentra el primer reconocimiento oficial por parte de la Iglesia de la existencia de diversidad de culturas (cf. AAS 36 [1944] 207-211). Años más tarde, en la encíclica misionera 
de la Iglesia por la cultura, en la manera de abordarla y de entender su relación con la fe cristiana. En la constitución Gaudium et Spes se encuentra -por primera vez en un documento de la mayor relevancia- una clara definición de lo que es la cultura desde una perspectiva más antropológica y sociológica; es decir, como un fenómeno plural:

“... se sigue que la cultura humana presenta necesariamente un aspecto histórico y social y que la palabra 'cultura' asume con frecuencia un sentido sociológico y etnológico. En este sentido se habla de la pluralidad de culturas. Estilos de vida común diversos y escalas de valor diferentes encuentran su origen en la distinta manera de servirse de las cosas, de trabajar, de expresarse, de practicar la religión, de comportarse, de establecer leyes e instituciones jurídicas, de desarrollar las ciencias, las artes y de cultivar la belleza. Así, las costumbres recibidas forman el patrimonio propio de cada comunidad humana. Así también es como se constituye un medio histórico determinado, en el cual se inserta el hombre de cada nación o tiempo y del que recibe los valores para promover la civilización humana" (53).

Vemos pues que ya no solo en el ámbito de las ciencias sociales sino también en el eclesial, la idea de "cultura" se asocia con las formas compartidas del lenguaje, del pensar, del actuar, del sentir, del valorar, etc., que son características de cada sociedad o grupo humano. O, más técnicamente, con la actividad de atribuir significados a hechos y personas mediante signos, constituyendo así un sistema simbólico históricamente construido (6). No se trata de conocimientos o refinamientos que tendrían unas elites intelectuales o artísticas, sino de las formas o sistemas simbólicos que operan de manera subterránea en el discurrir cotidiano de la gente, dando lugar a lo que el Concilio llama "estilos de vida común". Tales sistemas simbólicos están en permanente reelaboración, en la medida que lo demandan las transformaciones sociales o lo exige la sensación de pérdida de sentido de los marcos de comprensión vigentes.

Es importante destacar, como lo ha hecho el filósofo y teólogo Andrés Tornos a la siga de sociólogos y antropólogos, que la cultura tiene una función identitaria: es decir, las culturas median o intervienen necesariamente en la adquisición de la identidad propia de cada individuo y colectividad humana. Además -sostiene Tornos- la cultura tiene una función epistemológica: es decir, mediante la cultura compartida los individuos llegan a tener por verdaderos y básicos unos conocimientos o

Princeps Pastorum de 1959, Juan XXIII afirmaría que la Iglesia "no se ata a ninguna cultura, ni siquiera a la occidental y europea, con la cual está tan estrechamente ligada en su historia" (AAS 51 [1959] 833-864). Es decir, ya no solo avanza hacia una concepción más empírica de cultura, con el reconocimiento de múltiples culturas, sino que además se abre la posibilidad de percibir una Iglesia pluricultural.

(6) En esta línea va la definición de cultura del célebre antropólogo norteamericano Clifford GEERTZ, que podemos encontrar en su obra ya clásica La interpretación de las culturas (Ed. Gedisa, Barcelona ${ }^{5}$ 1992): "El concepto de cultura que propugno... es esencialmente semiótico. Creyendo con Max Weber que el hombre es un animal inserto en tramas de significación que él mismo ha tejido, considero que la cultura es esa urdimbre" (p. 20). 
representaciones que en otras culturas no se tienen por verdaderos y básicos (7). Las funciones identitarias y epistemológicas de las culturas están en permanente construcción; no son esencias innatas ni se dan de una vez para siempre.

Por un lado, entonces, la preocupación por la credibilidad del cristianismo tiene que considerar cómo ha de contribuir hoy la Iglesia en los procesos identitarios, en los que está en juego la dignidad de las personas y los pueblos. ¿De qué manera el Evangelio que ella ofrece puede impactar los sistemas de significación mediante los cuales se están reconstruyendo hoy las identidades? Dicho sea de paso, las instituciones educativas (colegios, universidades...) y los medios de comunicación social son claves, pero no los únicos ni tal vez las instancias más decisivas para "evangelizar las culturas". No basta tampoco decir que en el pasado la Iglesia ya realizó su aporte, dejando asentado un "sustrato católico barroco" en los pueblos latinoamericanos y que cualquier nueva síntesis identitaria que no considere ese sustrato, sería una suerte de desvío. Digámoslo una vez más: la Iglesia ha de tener en cuenta que las identidades están en permanente proceso de transformación (8) y que siempre es un desafío nuevo el contribuir en ese proceso. Y dada la diversidad de colectivos humanos con sus respectivas culturas, esa contribución no puede ser uniforme sino plural (9).

Por otro lado, el empeño por mostrar el carácter razonable del cristianismo con el fin de favorecer su credibilidad implica aceptar y contar con la pluralidad de representaciones de las verdades edificadas sobre los suelos de las distintas culturas. Hoy ya no hay excusa para desconocer los condicionamientos culturales de acceso a la verdad, tanto del evangelizador como del evangelizado. De hacerlo, se sigue

(7) Cf. Andrés Tornos, Inculturación. Teología y Método, Editorial Descleé de Brouwer/Universidad Pontificia Comillas, Madrid 2001, pp. 31-42.

(8) Sobre los cambios en la identidad de nuestro país, véase la obra de Jorge LARRAín: Identidad Chilena, LOM ediciones, Santiago 2001.

(9) En mi tesis doctoral intenté comprender, desde la perspectiva semiótica, la principal diferencia entre el discurso de Juan Luis Segundo con el de otros representantes de la teología de la liberación (en particular, con el de Gustavo Gutiérrez): es decir, el papel atribuido a los "pobres" ("beneficiarios" en Segundo; "protagonistas" y "beneficiarios" para Gutiérrez). Allí pude establecer que esa diferencia estaba condicionada por los interlocutores del discurso de cada uno de estos teólogos, por las comunidades eclesiales a las que estos acompañaban. Para Segundo, sus interlocutores son comunidades cristianas de clase media urbana; para Gutiérrez, comunidades cristianas constituidas por gente del pueblo pobre. En la tarea de acompañar a estas comunidades de distinta condición social, ambos teólogos han pretendido ayudar a sus miembros a configurar su identidad y a enfrentar los cambios que todos (clase media y pueblo pobre) parecen percibir como necesarios, a la luz de la palabra de Dios. Y, al hacerlo, da la impresión de que no pueden sino situar a sus interlocutores en la posición de "protagonista". Porque, en definitiva, cuando una teología quiere ser ayuda para que se consolide la identidad (cultural y cristiana) de un colectivo humano y para que se enfrenten los cambios percibidos como necesarios, la "dignidad" que está en juego para ese colectivo implica que se les sitúe como sujetos de su propia historia. Hay, pues, pluralismo dentro de la teología de la liberación, que se debe a la diversidad de colectivos humanos (y sus respectivas culturas) en la que dicha teología se ha fraguado; pluralismo que es necesario reconocer e incluso promover para el enriquecimiento de todos en la Iglesia (Cf. Fernando Verdugo, Relectura de la Salvación cristiana en Juan Luis Segundo. Estudio de las mediaciones culturales subyacentes a su soteriología de los años ochenta, Tesis doctoral, Universidad Pontificia Comillas, Madrid 1996/Anales de la Facultad de Teología, Vol. LIV, Pontificia Universidad Católica de Chile, Santiago 2003; Cap. IX, apartado 3: "Pluralidad de teologías de la liberación"). 
corriendo el riesgo de desprestigiar al propio Evangelio, de hacer que el mensaje de la Iglesia aparezca como inadecuado, intolerante o desfasado. No se trata de renunciar a la exigencia del Evangelio y su Verdad, sino de considerar cómo puede ser interpretado y acogido desde el suelo cultural del otro (10).

Para responder a esa interrogante, considero que el análisis cultural aparece cada vez más como un requisito imprescindible para llevar a cabo la tarea de evangelizar al hombre y sus culturas, para favorecer la credibilidad del cristianismo y, en definitiva, llegar a una genuina inculturación del mismo. Aun cuando la Iglesia católica, sobre todo a partir del Concilio Vaticano II y de la Exhortación Apostólica Evangelii Nuntiandi, haya dado un giro decisivo al darle importancia a lo cultural, ello no significa que haya puesto igual énfasis en la preocupación por analizar correctamente las diversas culturas. Es cierto que el Papa Juan Pablo II hace tiempo afirmó que la acción evangelizadora, en general, y la catequesis, en particular, "procurará conocer estas culturas y sus componentes esenciales; aprenderá sus expresiones más significativas, respetará sus valores y riquezas propias. Solo así se podrá proponer a tales culturas el conocimiento del misterio oculto y ayudarles a hacer surgir de su propia tradición viva expresiones originales de vida, de celebración y de pensamiento cristianos" (Catechesi Tradendae, 53). Sin embargo, considero que el uso de métodos de análisis que ayuden a "conocer las culturas" no se ha convertido en un hábito teológico y menos pastoral, no ha llegado a ser parte integrante del cumplimiento del mandato del Señor de llevar a todos el mensaje de la salvación, de hacerlo creíble en los diversos contextos.

Hace algunos años, de visita en nuestro país, Andrés Tornos sostenía que "el esfuerzo por analizar [las culturas] es lo único que permite superar las generalidades, o si la espontánea intuición de uno le aporta algo más que generalidades, entonces los lenguajes de análisis le permiten explicar sus intuiciones, contrastarlas, enriquecerlas y transmitir todo lo alcanzado" (11). Además, nos precisaba la doble finalidad del análisis para quien se interesa por lo cultural desde una perspectiva teológica y pastoral. Por un lado, se trata de llegar a "hablar con la gente" perteneciente a una determinada cultura o subcultura, o por lo menos "de caer en la cuenta de cómo habla la gente, cuáles son para ella los signos del bien, del sufrimiento, del mal, cuáles son los signos del poder y dónde se quiere estar respecto a él, qué signos tienen respecto del poder bueno y del poder malo, etc." (12); es decir, "entrar en los sistemas cognitivos y expresivos de la gente", percibir cómo organiza esa gente sus vidas y orienta su acción. Y, por otro, suponiendo que lo anterior no es por mera curiosidad, el análisis cultural debiera llevarnos a "poner la fe en los términos de la gente" (13).

Poner la fe en los términos de la gente implica volver a "producir" la verdad o las verdades fundamentales del Evangelio, atendiendo siempre a los procedimientos

(10) Para este tema, sigue siendo enormemente sugerente la obra de Claude GEFFRÉ, El cristianismo ante el riesgo de la interpretación, Ed. Cristiandad, Madrid 1984.

(11) Andrés Tornos, "La perspectiva del análisis cultural, en Arroyo, G., Silva, J., Verdugo, F., Por los caminos de América... Desafíos socioculturales a la Nueva Evangelización, Ed. Paulinas, Santiago (Chile) 1992, p. 213.

(12) Ib., p. 211

(13) Ib., p. 213 
mediante los cuales se construye la verdad en el grupo humano en el que se está inserto, con el que se dialoga o se quiere evangelizar. En ese proceso de producción de la verdad tendrá lugar lo que los sociólogos interaccionistas llaman la "negociación de significados", de modo que ella aparezca como relevante, oportuna para la existencia humana. Proponer una verdad implica, pues, preguntarse previamente cómo elabora el interlocutor una verdad en tanto que verdad, cuáles son los procedimientos mediante los cuales construye y tiene algo por verdadero y bueno; implica, luego, preguntarse cómo decir-verdad (veridicción), de modo que esa verdad sea acogida por quien la recibe como una buena noticia en la que se puede confiar.

Llevar adelante el proceso descrito, es la tarea que la Evangelii Nuntiandi asigna a las Iglesias particulares. Estas, dice la Exhortación apostólica de Pablo VI, "profundamente amalgamadas no solo con las personas, sino también con las aspiraciones, las riquezas y límites, las maneras de orar, de amar, de considerar la vida y el mundo que distinguen a tal o cual conjunto humano, tienen la función de asimilar lo esencial del mensaje evangélico, de trasvasarlo sin la menor traición a su verdad esencial, al lenguaje que esos hombres comprenden, y, después, de anunciarlo en ese mismo lenguaje" (63). Ahora bien, dada la complejidad de los fenómenos culturales -sobre todo en el contexto actual donde lo local está impactado por lo global- y la necesidad de realizar un discernimiento teológico de ellos, el aporte que una la Facultad de Teología puede ofrecer a las Iglesias particulares es insustituible y urgente.

En síntesis, hacerse cargo del tema de la credibilidad del cristianismo supone algo más que una mera sensibilidad por lo cultural. Considero que el quehacer teológico, en cualquiera de sus disciplinas o secciones, si desea favorecer hoy la credibilidad del cristianismo necesita de una mínima capacidad de acceso a los supuestos que se comparten o "negocian" entre los interlocutores sociales. Es necesario aprender a desentrañar las mediaciones culturales que operan en los procesos de construcción de identidades y en las maneras de decir o acoger una verdad. Hay que aprender a sacar a luz las disciplinas de pensamiento y lo que la semiótica llama los "procedimientos de veridicción" (14). Es cierto que son muchos los métodos de acceso a lo cultural (15). Con todo, considero que el análisis semiótico de la cultura, mediante el instrumental que ofrece, tiene la ventaja de permitirnos encontrar respuesta a preguntas como las siguientes: ¿en virtud de qué "signos" se identifica algo como verdad y, por tanto, como creíble en un determinado contexto? ¿Qué conjunto de reglas o "códigos" hacen que los signos o sistemas de signos que se utilizan

(14) "El análisis semiótico no intenta decir la 'verdad' de un discurso, sino que se interesa por la veridicción, es decir, por el conjunto de procedimientos por los que un discurso construye o produce su verdad" (J. C. GIROUD - L. PANIER, "Semiótica. Una práctica de lectura y de análisis de los textos bíblicos", Cuadernos Bíblicos N 59, Ed. Verbo Divino, Estella 21991, p. 34; véase también Greimas, A. J., Courtés, J., art. "Véridiction", en Sémiotique. Dictionnaire raisonné de la théorie du langage, Hachette, Paris 1993.).

(15) Robert SCHREITER, en Constructing Local Theologies, nos presenta diversas aproximaciones para comprender las culturas, señalando sus ventajas y desventajas, sobre todo con vistas a la construcción de teologías locales: la funcionalista, las ecológicas y materialistas, y la estructuralista (S. C. M. Press, London 1985; Orbis Books, Maryknoll, New York ${ }^{3} 1993$, pp. 45-49), inclinándose él mismo por la aproximación semiótica a la cultura (pp. 49-56). 
puedan ser portadores de mensajes tenidos por verdaderos y relevantes? ¿Cuáles son los "paradigmas" que, consciente o inconscientemente, se privilegian en una determinada cultura, de modo que el Evangelio, en interacción dinámica con ellos, aparezca efectivamente como buena noticia?

La tarea de hacer creíble la fe cristiana no sustituye, por cierto, la iniciativa, la gracia de Dios; pero contribuye a que, parafraseando a San Pablo, no nos avergoncemos de ella y sepamos en quién tenemos puesta nuestra fe (Cf. 2 Ti 1,12). Una expresión descontextualizada de la fe puede ser vergonzosa e incluso opacar el objeto de nuestra fe: la gracia que Dios "nos dio desde toda la eternidad en Cristo Jesús" (2 Ti 1,9).

Por último, me atrevo a afirmar que es en la vida cotidiana donde de hecho se decide lo que se tiene por verdadero, lo que es creíble y humanamente practicable. Es el ámbito en el que discurre la mayoría de la gente y los creyentes donde principalmente se negocian significados y se adopta lo que mejor se ajusta a la experiencia que se tiene de la realidad. O mejor dicho, mediante los significados que circulan en la vida cotidiana la mayoría de los seres humanos, incluidos los creyentes en Jesucristo, construyen, dan sentido y organizan su experiencia de la realidad. Por tanto, en un Seminario como el que estamos comenzando es necesario indagar cómo resuelven o abordan creyentes y no creyentes "de a pie" los desafíos que hoy plantea el nuevo escenario.

Pero eso, entiendo, es parte de la tarea que le corresponde a los demás actores de este Seminario Interno de la Facultad de Teología (16).

\section{RESUMEN}

A modo de introducción al Seminario Interno de Profesores de la Facultad de Teología de la PUC, el autor recuerda, en primer lugar, lo que suele entenderse por "credibilidad" y, también, algunos de los criterios generales por los cuales algo o alguien adquiere la calidad de creíble (el que sea razonable, relevante o significativo, capaz de producir gozo estético, etc.). Luego, en la parte más extensa, el autor vuelve a llamar la atención sobre la necesidad de atender a los condicionamientos culturales de la fe cristiana. La fe, al igual que toda experiencia humana, está culturalmente mediada. Tomar conciencia de ese hecho y asumir las consecuencias metodológicas es indispensable para una teología que quiere poner atención a los desafíos actuales y contribuir así a la credibilidad del cristianismo.

\section{ABSTRACT}

This article reprints the introductory session to the internal seminar of lecturers from the Faculty of Theology of the UC. In the first place, the author sums up what is usually understood by 'credibility' and the general criteria through which something or someone gains credibility (being reasonable, relevant or significant, capable of producing aesthetic pleasure). Then the

(16) Por mi parte, como fruto de una ponencia que hice a la Comisión Pastoral de la Conferencia Episcopal de Chile, publiqué lo que considero algunos de los principales desafíos socioculturales que hoy tiene la Iglesia en el país ("La Iglesia y los cambios culturales", Mensaje 512 [septiembre 2002] 12-16). 
author deals at length with the need to take notice of the cultural conditionings of the Christian faith. The faith, the same as any other human experience, is culturally mediated. It is crucial for theology to become aware of this fact and come to terms with its methodological consequences, if it wants to face current challenges and so contribute to the credibility of Christianity. 